\title{
A Systemic Approach to Multi-Party Relationship Modelling
}

\author{
Anshuman B. Saxena ${ }^{1,2}$ and Alain Wegmann ${ }^{1}$ \\ ${ }^{1}$ Systemic Modeling Laboratory, EPFL Station 14, 1015 Lausanne, Switzerland \\ ${ }^{2}$ TATA Consultancy Services Innovation Labs, EPIP 96 Whitefield, 560066 Bangalore, India \\ anshuman.saxena@epfl.ch, anshuman.saxena@tcs.com, \\ alain.wegmann@epfl.ch
}

\begin{abstract}
Socio-economic systems exist in a wide variety of activity domains and are composed of multiple stakeholder groups. These groups pursue objectives which are often entirely motivated from within their local context. Domain specificities in the form of institutional design, for example the deregulation of Public Utility systems, can further fragment this context. Nevertheless, for these systems to be viable, a management subsystem that maintains a holistic view of the system is required. From a Systems perspective, this highlights the need to invest in methods that capture the interactions between the different stakeholders of the system. It is the understanding of the individual interactions that can help piece together a holistic view of the system thereby enabling system level discourse. In this paper we present a modelling technique that models industry interactions as a multi-party value realization process and takes a Systems approach in analyzing them. Every interaction is analyzed both from outside - system as a black box and from within - system as a white box. The design patterns that emerge from this whole/composite view of value realization provide the necessary foundation to analyze the working of multi-stakeholder systems. An explicit specification of these concepts is presented as Regulation Enabling Ontology, REGENT. As an example, we instantiate REGENT for the urban residential electricity market and demonstrate its effectiveness in identifying the requirements for time-based electricity supply systems.
\end{abstract}

Keywords: Value modelling, Ontology design, Industry de-regulation.

\section{Introduction}

Socio-economic systems are composed of multiple stakeholder groups. These groups can be viewed as bound together in one system by virtue of their membership to a shared relationship - the interactions between individual groups being different instances of this relation. Nevertheless, the diversity and plurality of stakeholder beliefs encourage individual stakeholder groups to pursue objectives entirely motivated from within their local context. In addition, domain specificities in the form of institutional design, for example the unbundling of large, vertically integrated Public Utilities into lean, efficient and more focused entities can further fragment this context [3]. Public Utilities [19], such as electricity, telecommunication, transportation, posts, gas and water supply, are most representative of such 
restructuring. From a management perspective, such unbundling results in the dissolution of the high level management structures which, in the pre-deregulated era, were responsible for the end-to-end delivery process. A deregulated industry is, instead, composed of multiple smaller management structures - each restricted in scope to some specific aspect of the industry. The absence of a holistic industry wide management structure makes deregulated industries vulnerable to systemic failure. For these unbundled entities to work as a viable whole [5], a management subsystem that maintains a holistic view of the constituent sub-systems is required. In the Public Utility domain, such a system wide management structure is usually available in the form of Industry Regulator. Nevertheless, modern regulatory systems need to go beyond the usual concerns of price, quality, output and access, and invest in schemes that capture the interactions among the stakeholders of the industry. Understanding the individual interactions help piece together a holistic view of the industry, thereby allowing the regulator to devise well informed interventions aimed at strengthening the four major quality systems constituting sustainable development - people, economic development, environment and availability of resources [27].

To address these challenges, we invoke the notion of value and model every relationship in an industry as a set of value realization processes. Value is a qualitative concept and, thus, well suited for an interdisciplinary discourse. Taking a Systems perspective, we analyze the value realization process both at the industry level and at the level of individual stakeholders within the industry. Two important design patterns emerge from this whole/composite view of value exchange: any value created in an industry has an associated supplier and adopter; a supplier of one set of value is an adopter of some other set of value. These design patterns form the basis for formalizing the concepts required to explain multi-party relationships in an industry.

This paper is an attempt to provide an explicit specification of these concepts as ontology. The ontology will provide regulators with a standard representational vocabulary with which they can document the material and information interplay between the different stakeholders of an industry. It is the abstraction of industry specific configuration details as shared pan-industry concepts that will facilitate the knowledge-level communication among the community of regulators, thereby enabling more effective and speedy sharing of regulatory best practices. Section 2 provides a brief overview of Systems thinking approach and presents a Systems perspective of the de-regulated electricity supply industry. Section 3 explores the notion of value in greater detail and introduces the concepts of resource and feature as building blocks of the value realization process. Section 4 describes the Regulation Enabling Ontology, REGENT, in detail, highlighting the different design choices that were made during the development of REGENT. Section 5 instantiates REGENT for the urban residential electricity market and, as an example, demonstrates its effectiveness in establishing regulatory oversight. Section 6 presents some related work in this field. The paper concludes with future work directions in Section 7.

\section{A Systems Perspective of Industry}

A Systems approach to understanding the relationship between the stakeholders of an industry allows taking a holistic view of the industry and analyzing how these relationships influence one another in the context of the overall well being of the 
industry. This is particularly useful for deregulated industries where management structures only exhibit knowledge about local relationships and the relevance of these relationships to the entire system remains largely unexplored. For a regulator to act as a true custodian of the industry, it is important that it has the complete knowledge about the different interactions that occur in an industry and the bearing these relationships may have on the overall working of the industry. To further illustrate the affect of deregulation on the overall management of the industry, we use the visual semantics of SEAM to analyze the evolution of Electricity Supply Industry (ESI).

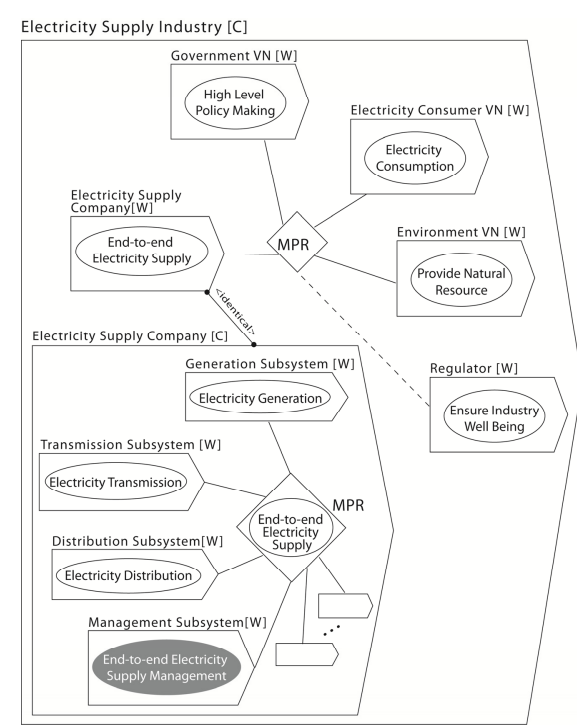

Fig. 1. Pre-deregulated ESI

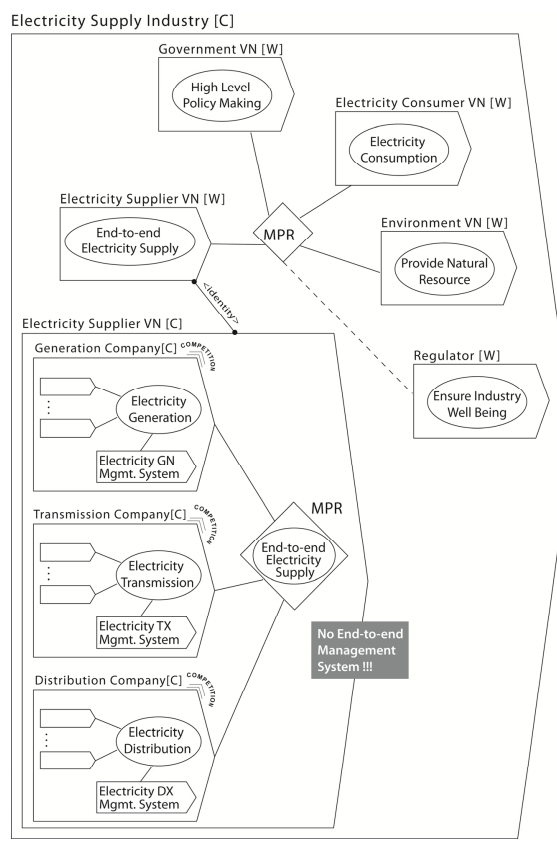

Fig. 2. Deregulated ESI

SEAM is a set of Systemic Enterprise Architecture Methods [25] that exploit the principles of General Systems Thinking (GST) [26]. GST advocates that the component parts of a system can be best understood in the context of relationships with each other and with other systems, rather than in isolation. An important way to fully analyze a system is to understand the part in relation to the whole. SEAM represents any perceived reality as a hierarchy of systems. Each system can be analyzed as a whole [W] - showing its externally visible characteristics or as a composite [C] - showing its' constituents as a set of interrelated parts. When applying SEAM to an industry, two main aspects are analyzed: (1) How different stakeholders cooperate together to achieve some common objective; these groups of stakeholders are referred to as value network, VN. (2) How these value networks interact within an industry; these interactions are referred to as Multi-Party Relationship, MPR. The visual syntax of SEAM includes block arrows for systems, annotated ovals for externally visible properties, diamonds for relations, simple lines for active 
participation to a relation, dashed lines for pseudo participation to a relation and rounded end-point lines for emphasizing the identical nature of modelling elements.

Figure 1 presents a SEAM depiction of a pre-deregulated Electricity Supply industry. The four prominent entities that engage in the activities of this industry are the Electricity Supply Company (ESC), Electricity Consumer VN, Government VN and the Environment VN. When viewed as a whole, the ESC [W] exhibits the overall responsibility of maintaining an end-to-end supply of electricity - from generation to distribution. When viewed as a composite, the ESC [C] reveals its' constituent subsystems. ESCs can have different architectures. Nevertheless, for these subsystems to work as a viable whole, each ESC has some form of management subsystem [5] that oversees the end-to-end delivery process.

Figure 2 presents a SEAM depiction of a deregulated Electricity Supply Industry. The vertically integrated ESC of the pre-deregulated era stands unbundled into independent Generation, Transmission and Distribution Companies. The presence of multiple such companies constitutes competition, and provides the Electricity Consumer VN the choice to buy electricity from one Generation Company, get it transmitted through some other Transmission Company and receive the end supply service from yet another Distribution Company. These three companies when put together represent the Electricity Supplier VN. From a management perspective, each of these companies is controlled by an independent management sub-system which is strictly limited to its' part of industry operations, e.g. generation, transmission or distribution. Unlike the pre-deregulated era, there exists no end-to-end electricity supply management system that can be held responsible for the overall supply.

\section{Multi-party Relationship}

An industry is a complex composition of diverse stakeholder groups. Suppliers are primarily concerned about issues related to market share, profit and return-oninvestment; consumers are concerned about cost, availability, reliability and ease-ofuse; governments are concerned about collective welfare, institutional relevance and political indispensability; and the issues of interest from an environment point of view include habitat and climate related ecological concerns. To realize the benefits of Systems approach in analyzing the different facets of an industry, it is important to first identify a unifying concept that can act as a generic platform for the interdisciplinary discourse required in an industry. In this paper we exploit the notion of value as the unifying concept and treat the above mentioned stakeholder concerns as context specific manifestations of the value concept.

Based on the analysis presented in [19], we define value as the tangible or intangible effect accrued by a stakeholder through the consumption or trade of a service or good. The notion of value is at the heart of MPR modeling. Stakeholders aspiring for a common set of value are grouped together as a VN. MPR models industry interactions as a value realization process between VNs. VNs exchange resources, material and information. Any resource addition to the $\mathrm{VN}$ affects the stakeholders of the VN either in a favorable way, realizing positive value, or in an unfavorable way, realizing negative value. Figure 3 depicts MPR as a bi-directional value realization process between the different VNs in an Electricity Supply Industry. 


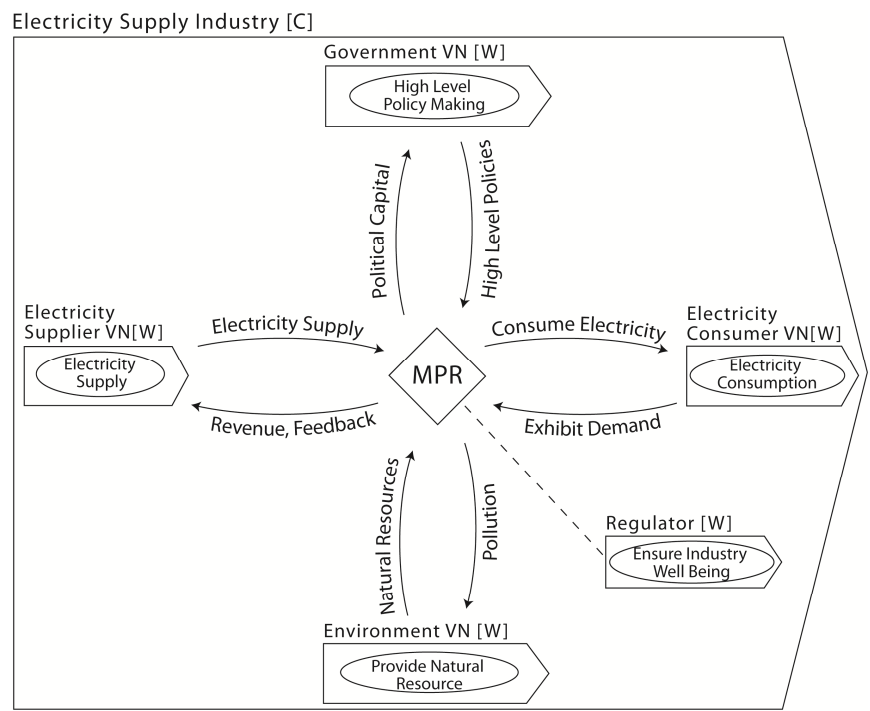

Fig. 3. Bi-directional value realization in MPR

Value is a subjective notion, dependent exclusively on stakeholder perceptions. An effect welcome by some stakeholders may be completely rejected by others. For example, time based electricity pricing schemes where a consumer can pay less for off peak electricity usage is perceived by many as a positive value as it provides an opportunity to reduce electricity bills by shifting workloads to low cost off peak durations. For others this may not be a welcome change as it results in increased night time activity in the neighbourhood. As a result it is desirable to explicitly specify the context in which a value is created, delivered or consumed. We accomplish this by introducing the concepts of resource and feature.

We follow the definition given in [4], where resources are defined as "... assets, capabilities, processes, and information" in control of the stakeholder. Thus resource can be considered as the contribution an individual stakeholder can bring to a VN. Feature on the other hand is a composite attribute which exists only at the VN level. Based on the resources available with the different stakeholders of a VN, the VN may exhibit different properties. These properties emerge from the different combinations between these resources, and are known as the features of the VN. For a given industry, an MPR identifies the different resources available with each VN, the set of possible features that may emerge from them and the value these features may bring to the other VNs. The same is presented in Figure 4. The use of the term enterprise in the figure is a more formal way of referring to stakeholders constituting a VN. The resource, feature and value concepts coupled with the GST inspired whole-composite view of value exchange guides our ontology design activity. Two important design patters emerge from this combination.

D1. For every value created in an industry there exists a supplier VN and an adopter $\mathrm{VN}$

D2. Each VN in an industry acts as a supplier of one set of value and an adopter of another set of value 


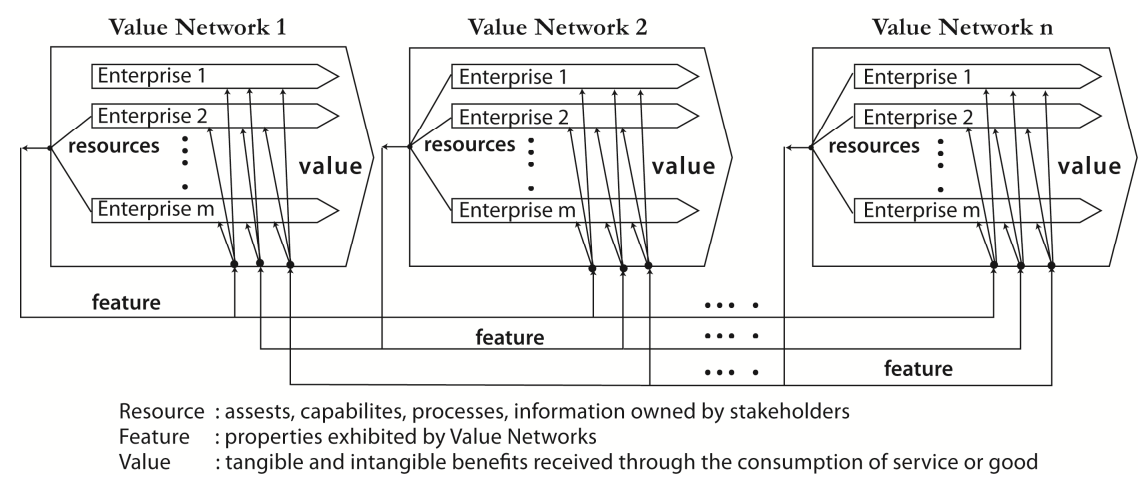

Fig. 4. The Resource-Feature-Value triune in MPR

Supplier and adopter are roles assigned to VNs while analyzing MPRs. The supplier role signifies ownership of resources required to create/produce and deliver the services or goods. The adopter role signifies ownership of resources required to consume the service or good thereby realizing the value advertised through the features of the service or good.

Design Patterns have their genesis in the field of architecture where they were first proposed as an architectural concept by Christopher Alexander [1]. These were later adopted in software engineering, and are defined as an artifact in the form of a construct, a model, a method or an instantiation, which is general enough to be reusable in solving commonly occurring problems [8]. In this paper we use these two design patterns as the basic constructs for formally specifying the knowledge required to formulate an overall understanding of any industry.

\section{REGENT: A Regulation Enabling Ontology}

As defined in [12], ontology is an explicit specification of a shared conceptualization. It is aimed at formalizing a specific view point that enables/enriches the discourse on some aspect of interest in the real world. The purpose of REGENT is to enable the discourse on industry regulation. Formalization of the concepts that constitute an industry and the relationships that hold among these concepts provides a common vocabulary with which regulators can represent their understanding of the industry. Such a standardized way of documenting information is particularly useful in promoting knowledge-level communication between the different industry regulators.

Various ontology languages exist to represent these concepts and relationships. The most prominent of these is OWL [24]. It is developed by the World Wide Web Consortium and consists of individuals, properties, and classes. Individuals represent the objects in the domain of interest, properties are binary relations on these individuals, and classes are interpreted as sets that contain these individuals. Our reference to concept and relationship maps to the notion of class and property in OWL. Individuals are instantiation of concept. OWL has three sub-languages: OWLLite, OWL-DL and OWL-Full. The expressiveness of OWL-DL falls between that of OWL-Lite and OWL-Full. It is based on Description Logics [2] which are a decidable 
fragment of First Order Logic and are thus conducive for automated reasoning. For this purpose we use OWL-DL as the language for specifying REGENT. The development of REGENT was done using the ontology development tool, Protégé [22]. The visualizations presented in this paper have been created using the OntoViz graphical plug-in in Protégé. In the following, we present our design choices for REGENT.

REGENT has two top level classes: Industryconcept class and ConceptSpacePartition class. IndustryConcept is the foundational class for all the concepts in an industry. It is based on the Resource-Feature-Value triune detailed in sub-section 2.3. ConceptSpacePartition is the class which subsumes the different viewpoints that can be useful in analyzing the set of concepts detailed in the IndustryConcept class.

\subsection{The IndustryConcept Class}

The IndustryConcept class formalizes the concepts of resource, feature and value. Figure 5 presents the taxonomy of the Resource class. The Resource class has two subclasses: Commercial and Operational. This refinement of the Resource class is a manifestation of the design pattern D2. As depicted in Figure 3, every value realization is a bi-directional process. We exploit the dual nature of $\mathrm{VN}$, i.e. the simultaneous role of a supplier of one value and an adopter of some other value, to classify the resources available with a VN. From an industry perspective, a product or service creation process has two parts - the operational process of bringing the service or good into existence and the commercial process of making it tradable [21]. The operational process is related to the supplier role of $\mathrm{VN}$; the supplier has complete control over this process. On the other hand, the commercial process is related to the adopter role of VN. It is aimed at making the service or good conducive for consumption and, thus, requires taking an adopter perspective. Accordingly, the set of resources in an industry can be divided into two - the ones required to realize the operational process, the RS_operational class, and the others required to realize the commercial process, defined as the RS_Commercial class.

We can further refine this classification by exploiting the insights of the supplier and adopter process. At the supplier end, bringing a service or good into existence entails two aspects - production and delivery. For instance, in the Electricity Supply Industry it is not sufficient for the electricity to be generated at the generation units, it is equally important that it is available at the prospective location of consumption. Operational resources that contribute towards the production of the industry offering are categorized as the RS_OP_Production class while the ones that contribute towards the delivery of the industry offering are categorized as the RS_OP_Delivery class. At the adopter end, realizing the benefits of the offering entails two aspects reception and consumption. For instance, the complementary nature of electricity requires the availability of electrical appliances to consume electricity. Commercial resources that contribute towards the consumption of the industry offering are categorized as the RS_CM_Consumption class while the ones that contribute towards the reception of the industry offering are categorized as the RS_CM_Reception class. Finally, based on their cognitive orientation a resource can be further classified as 
tangible and intangible. The leaf nodes of the taxonomy presented in Figure 6 refine the higher level RS_CM_* and RS_OP_* classes as RS_*_*_Tangible and RS_*_*_Intangible subclasses.

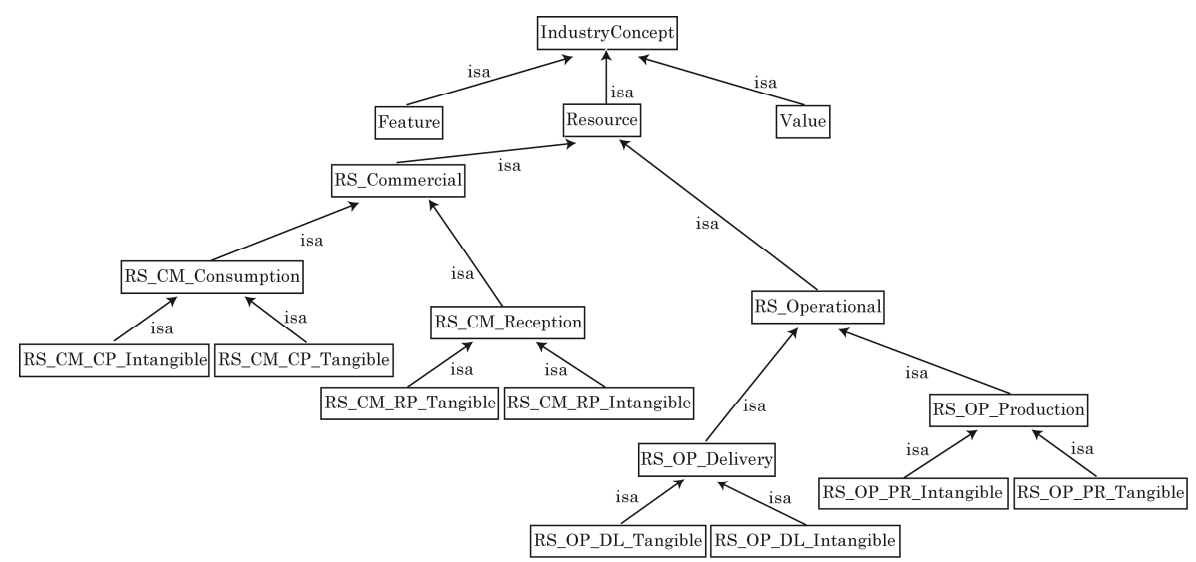

Fig. 5. Taxonomy of the Resource class

Figure 6 presents the taxonomy of the Feature class. The Feature class is a manifestation of the design pattern D1. As argued in [19], we do not treat value as an intrinsic characteristic of a product or service, and hence do not subscribe to the value chain metaphor [17] which is often interpreted to suggest that a value can be moved from the supplier to the adopter. The notion of supplier and adopter in D1 is to highlight the role of VNs in supplying resources that lead to the realization of some value at the adopter VN. Nevertheless, connecting resources directly to value will bypass an intermediate composition level where resources from different enterprises within a VN come together to define artifacts with some potential value content. This concept of composition is concretized in the Feature class. Features can, thus, be viewed as the potential value of a combination of one or more resources of a supplier VN. This potential value gets transformed into realized value when the adopter VN consumes the underlying artifact i.e. the industry offering. Thus feature and value differ only in the context of the observer. Feature expresses the view of the supplier of his product or service and value is the view of the adopter of the consumed product or service. This difference is captured as property constraints and is further detailed in Section 4.3.

From a taxonomy point of view, interpretation of features as potential value results in similar refinements of the Feature and Value classes. The taxonomy of the Feature class is presented in Figure 6. We posit that the Value class has a similar taxonomy tree hence do not present it separately. The following discussion on the specificities of feature refinement applies equally to the value concept.

The Feature class has two subclasses: FT_Utility and FT_Warranty. Utility and warranty are two concepts publicized as part of the Information Technology Infrastructure Library (ITIL) [16], developed by the UK's Office of Government Commerce (OGC) for Information Technology Services Management. Utility captures the functionality offered by a product or service and is informally 
interpreted as 'what the industry offering does'. On the other hand, warranty is the promise that a product or service will meet its' agreed requirements, informally interpreted as 'how the industry offering is done'. In the Requirements Engineering field, these are often termed as the function and non-functional requirements [9].

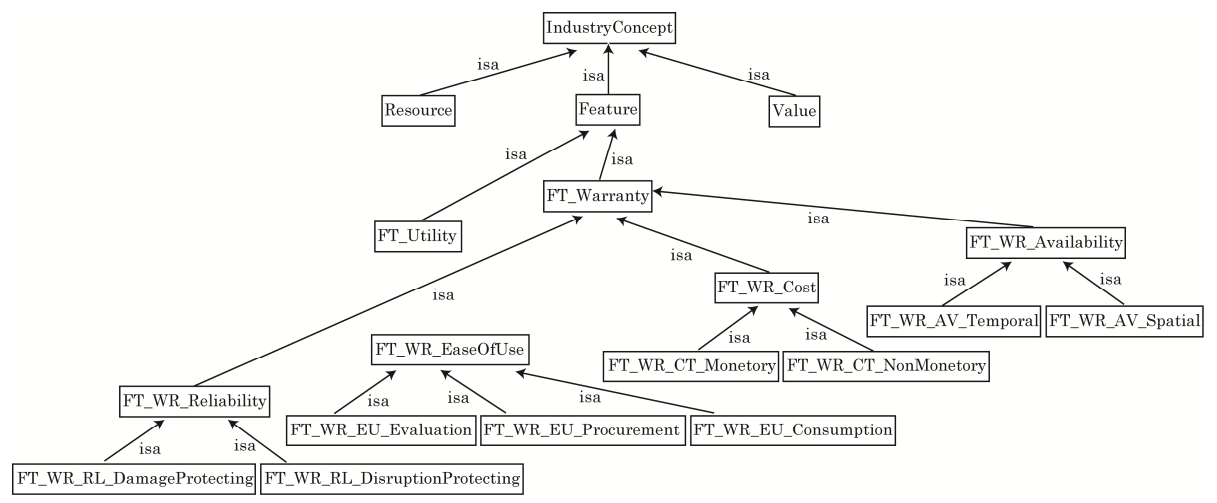

Fig. 6. Taxonomy of the Feature class

The utility of a service or good is usually well understood. It is the warranty aspect that is open to interpretation and is hence further refined. A warranty can be related to the availability, reliability, ease of use and cost of the service or good. The FT_WR_Availability class represents the attributes that capture the readiness of the service or good to be consumed by the adopter. The readiness can be both temporal, FT_WR_AV_Temporal class, and spatial, FT_WR_AV_Spatial class. The presence of electricity supply at the time and place of consumption will constitute the temporal and spatial availability of the service provided by the ECN. The objects of the FT_WR_Reliability class represent the appropriateness of the service or good for consumption. Appropriateness can be achieved by ensuring safeguards against disruptive failures, the FT_WR_RL_DisruptionProtecting class, and damaging failures, the FT_WR_RL_DamageProtecting class. For instance, the use of surge protector equipment can protect against slight variations in electricity supply but a line breaker would be required to stop the supply in the event of very high variations in supply. The FT_WR_EaseOfUse class represents the (in) convenience of evaluating - FT_WR_EU_Evaluation, procuring - FT_WR_EU_Procurement, and consuming FT_WR_EU_Consumption, a product or service. The FT_WR_Cost class captures the attributes that define the cost of the service or good. The cost can be interpreted both in monetary, FT_WR_CT_Monetary, and in non-monetary terms, FT_WR_CT_NonMonetary.

\subsection{The ConceptSpacePartition Class}

The taxonomy of the ConceptSpacePartition class is presented in Figure 7. As the name suggests, this class creates a partition on the set of concepts represented in the IndustryConcept Class. A partition imposes a certain view of the industry. The Enterprise subclass partitions the various concepts in an Industry along the well 
established boundaries of legal ownership and undertaking. For instance every resource in an industry is owned by some enterprise. Enterprise subclass is the default partition of the objects represented by Industryconcept class.

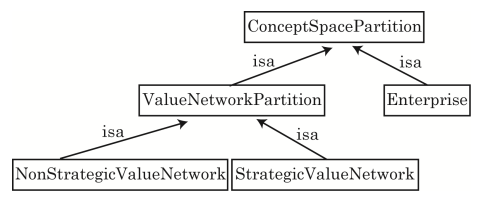

Fig. 7. The Taxonomy for ConceptSpacePartition Class

The ValueNetworkPartition subclass is a manifestation of the Value Network concept in SEAM. It relies on the default Enterprise class imposed partition on industry concepts. More specifically, the ValueNetworkPartition subclass partitions the various concepts in an industry along the common intent of the enterprises where these concepts originate. It is important to note that the absence of an explicit intent is also a commonality and, hence, can form a valid partition of the Industry concepts. As a result, the ValueNetworkPartition class is further subdivided into VNP_Strategic and VNP_NonStrategic. The strategic subclass refers to a partition that is based on some maximizing something - profit, welfare, power, etc. By contrast, the non-strategic subclass is blind and has no objective, no preferences, and no foresight, for instance the Environment [6].

\subsection{Property Constraints}

The properties that bind the different concepts in REGENT are depicted in Figure 8. Properties in OWL are binary relations constraining the interaction between any two classes. For any property connecting an object o1 to object 02 an inverse property can also be specified which connects object 02 with $\mathrm{o}$. In the following, we discuss these properties on a class by class basis. For the sake of clarity, words starting with upper case alphabet are class names and the same when written in lowercase represent objects of that class.

The objects in the Resource class are constrained through two properties. 1) The hasOwner property mandates that each resource is connected to some enterprise. To ensure the uniqueness of this relation we limit the property to have a single value i.e. each resource has only one owner. In OWL this is accomplished by setting the property characteristics as functional. The corresponding inverse property that connects an enterprise to its resources is the is OwnerOf property. The one-to-many nature of this relation is visually represented with an asterisk (*). An enterprise can own more than one resource. 2) The isProviderOf property links a resource to the feature it contributes. The corresponding inverse property that connects a feature to its constituent resources is the hasProvider property. Both of these properties represent a one-to-many relation - a resource can enable more than one feature and a feature can be enabled by more than one resource.

The objects in the Feature class are constrained through four properties. 1) The hasTransformationTo relation specifies the values that are realization of the features. 
The corresponding inverse property isTransformationFrom specifies the features that constitute the value. Both of these relations exhibit multiplicity - multiple features can aid a value creation and multiple values can be enabled by a feature. 2) The hasSupplier relation specifies the supplier value network for a feature. This is a single value relation which restricts each feature to have a unique supplier. The same is imposed by setting the functional characteristic of this property. The corresponding inverse property, isSupplierOf, is a multi-valued relation. A value network can be a supplier of more than one feature. 3) The hasProvider relation is already discussed above. 4) The hasAdopter relation specifies the adopter value network for a feature. The corresponding inverse property, isAdopterOf, specifies the set of features that a value network adopts. Both of these are multi-valued properties - a value network can adopt multiple features and a feature can be adopted by multiple VNs.

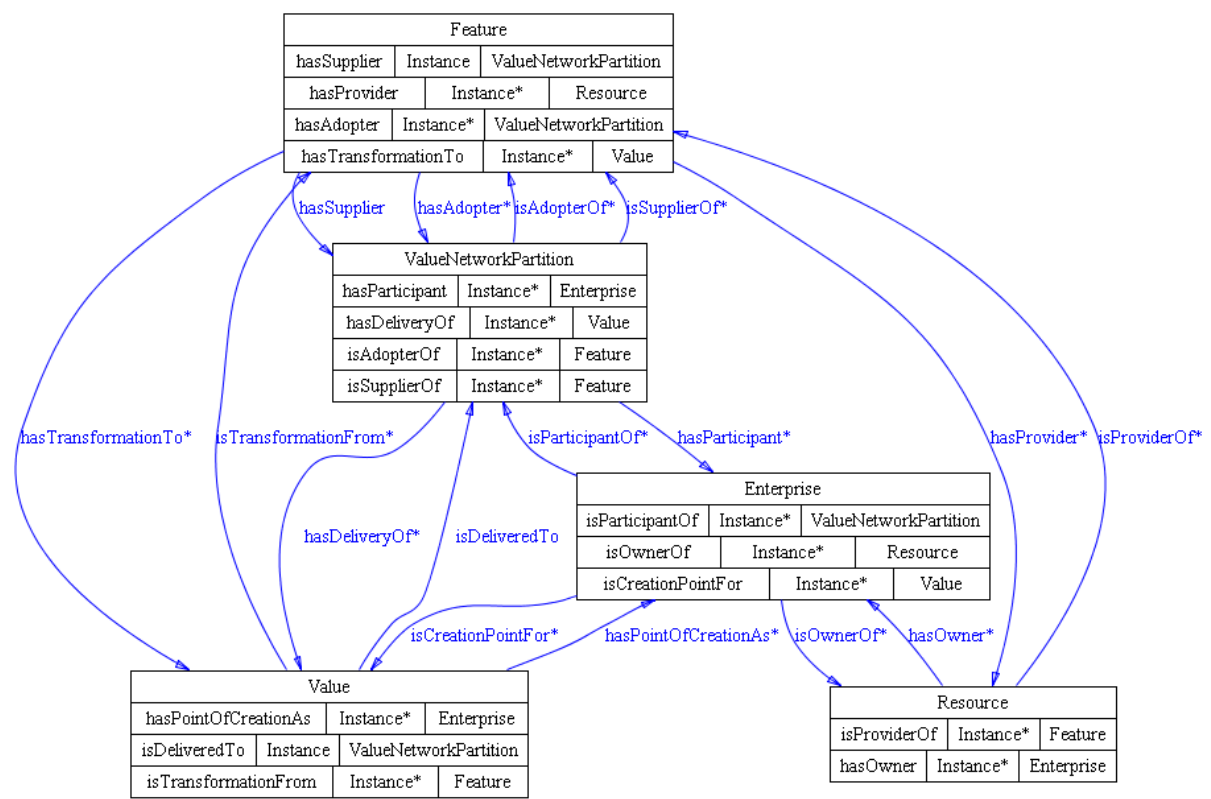

Fig. 8. A visual representation of properties constraining REGENT concepts

The objects in the Value class are constrained through three properties. 1) The isDeliveredTo property specifies the value network where a value is realized. This is a single value property; a value is closely associated to the perception of the consumer and, is hence, unique to the value network. We do this by setting the functional characteristic of the property. The corresponding inverse property, hasDeliveryOf, specifies the value that a value network consumes. 2) The hasPointOfCreationAs property specifies the precise enterprise which consumes this value. Again, consumption is unique to an enterprise; hence, this property is a single-valued function. The corresponding inverse property, isCreationPointFor, identifies all the values that are consumed by an enterprise. This is a multi-valued property. 3) The isTransformationFrom property has been detailed earlier. 
In addition to the properties exhibited by the Feature, Resource and Value class. There exists an additional relation between the objects of the Enterprise class and the objects of the ValueNetworkPartition class. The property isParticipantOf identifies the value network to which the enterprise participates. To highlight the fact that an enterprise when part of two value networks does so in different roles, we model this relation as a single-value property - setting its functional characteristic. The corresponding inverse property, hasParticipant, is a multi-valued property and identifies all the enterprises that are members of a $\mathrm{VN}$.

\section{The Case of Urban Residential Electricity Supply}

In this section, we use REGENT to provide a systematic view of the Urban Residential Electricity Supply Industry (URESI). Details about the URESI were gathered from various reports [23] [14], best practices [15], guidelines [18], national regulations [10] and personal communication with Industry representatives. The later was done through a consultation meeting, 'The Role of IT in Regulatory Governance', held on December 05, 2009 at TATA Consultancy Services Ltd., Lucknow India.

We begin by identifying the different stakeholders in a URESI. Stakeholders with common objectives, or lack of objective, are grouped into same Value Network. Four VNs emerge from this exercise: The Economic Value Network (ECN) that represents enterprises with primarily economic motivation, Social Value Network (SCN) that represents enterprises with primarily social motivation, Environmental Value Network (EVN) that represents non strategic enterprises and Government Value Network (GVN) that represents the collective welfare as the overriding motivation. The enterprises constituting the ECN are Generation Company, Transmission Company and the Distribution Company. The enterprise constituting the SCN is the Urban Household. The enterprises constituting the ENV are Climate and Habitat. Climate represents the macro level aspects of the environment while habitat represents the micro level aspects of our immediate surroundings. ECN and SCN are generalizations of the Electricity Supplier Value Network and the Electricity Consumer Value Network mentioned in the Sections 2 and 4.

\subsection{Resource Identification}

For each of these VN, we take a commercial and operational view of the value exchange and identify the tangible/intangible resources that aid the production/delivery of the $\mathrm{VN}$ offering and the reception/consumption of the counter offering from other VNs. These resources along with the related Enterprise and Value Network are listed in Table 1.

\subsection{Feature Identification}

Every VN in an industry contributes some service or good to other VNs in the industry. As described in Section 2.4.1, a VN offering can be detailed along the utility and warranty dimensions. Table 2 lists the utility and warranty details of the VN offerings in the Urban Residential Electricity Industry. 


\subsection{Value Identification}

Every $\mathrm{VN}$ in an industry receives some value in return to his contribution to the Industry. Value can either be positive or negative, solicited in the case of strategic VNs or unsolicited in the case of non-strategic players. Table 3 lists the utility and warranty of the different value created in the Urban Residential Electricity Supply Industry, the VNs that adopt these value and the enterprises in the adopter VN where these value are realized.

Table 1. List of Resource identified in URESI

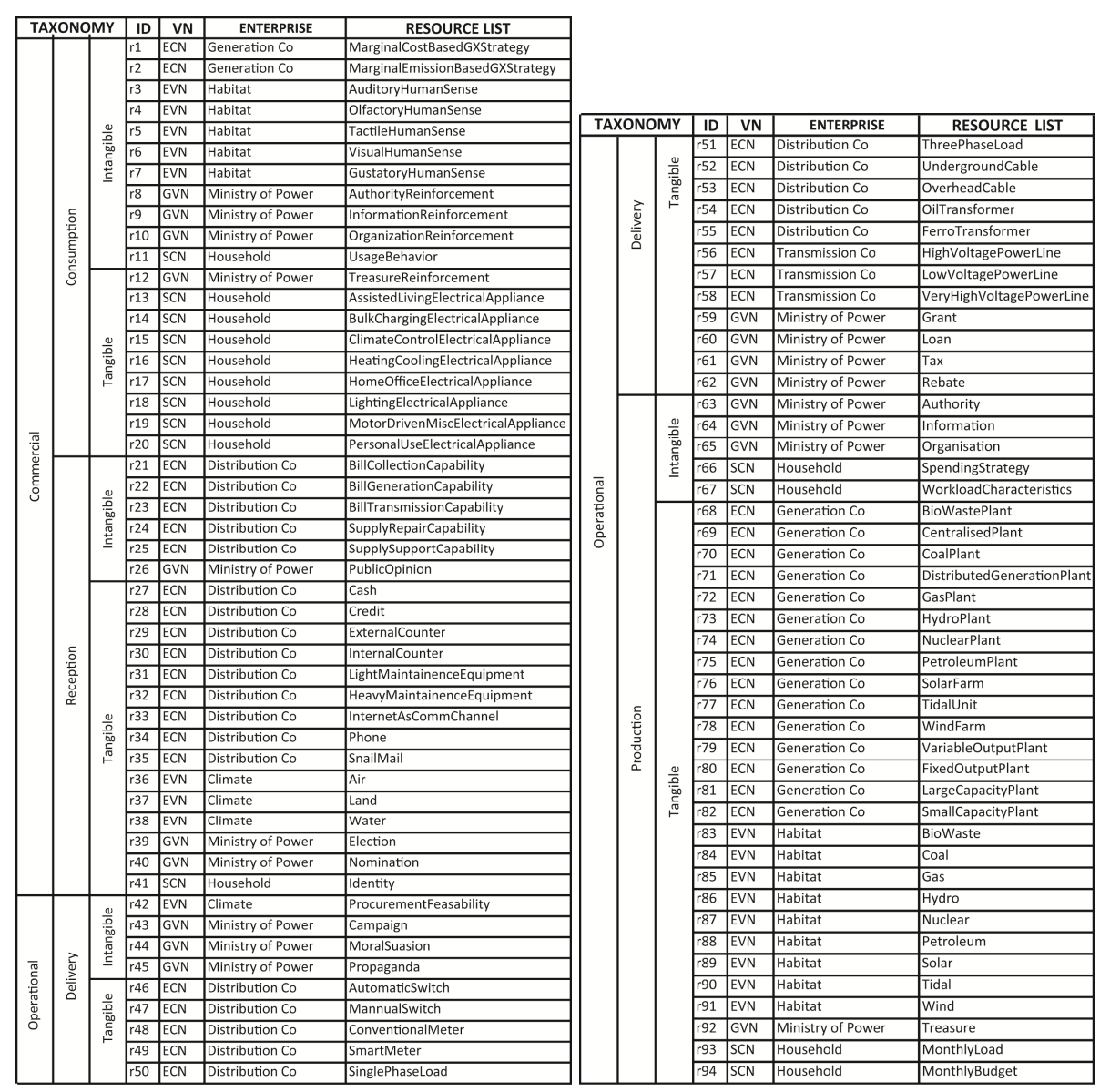

\subsection{Establishing Regulatory Oversight}

Balancing the supply and demand for electricity is central to the proper functioning of an electricity grid. The demand, however, tends to exhibit time sensitivities with more electricity required during specific times of the day or year, for example increased lighting requirements during the night and higher climate control needs during peak 
winter/summer season. In the absence of efficient large scale electricity storage techniques such variability in demand can only be met through flexible generation capabilities. Not all generation units support variable output. For example, nuclear power plants must be run at close to-full capacity at all times whereas production from other sources such as wind and solar, though inherently variable in nature, remains hard to predict. Further, the cost of electricity production varies from one type of generation unit to another. Generation Company operates these units in an increasing order of marginal costs (r1). Thus increased generation required to meet higher demands (peak hours) results in a higher per-unit cost of electricity. Similarly, during periods of low demand (off-peak hours) generation units with high marginal costs are cycled down resulting in a lower per-unit cost of electricity. Installation of smart meters (r49) allows the Distribution Co. to extend its billing capability (r22) and help the ECN introduce time of use (ToU) electricity pricing tariffs (f44). ToU presents economic incentives to enterprises in ECN and SCN alike. Electricity suppliers can increase profits by charging a higher per-unit cost during peak hours and consumers can minimize their bill (f48) by moving their time insensitive workloads (f35) to off-peak hours when the per-unit cost is low. The sensitivity of households to electricity bill is a function of their monthly budget (r94) and spending strategy (r66). Any attempt by households to move electricity workloads to off-peak hours is limited to the rescheduling of time insensitive workloads (f35) which in turn depends on the availability of requisite electrical appliances $(\mathrm{r} 14,19)$ and batch oriented workload characteristics (r67).

Table 2. List of Feature identified in URESI

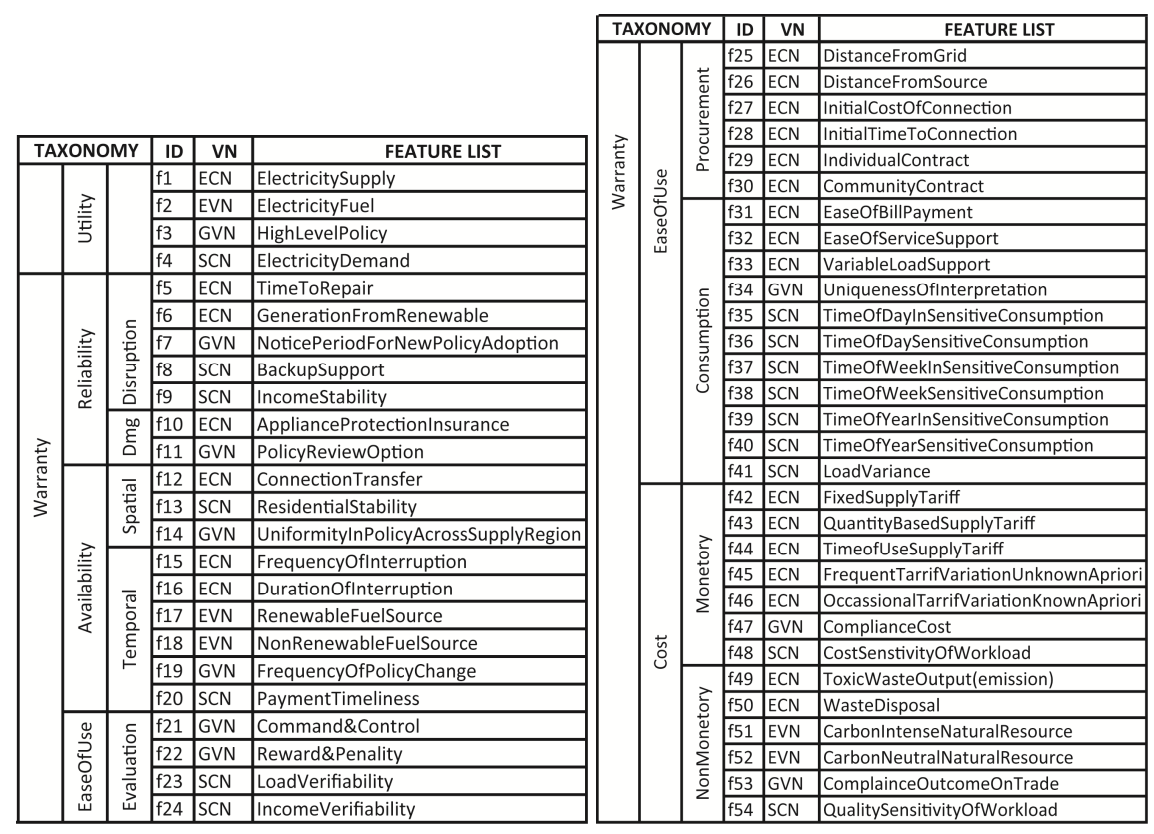


Table 3. List of Value identified in URESI
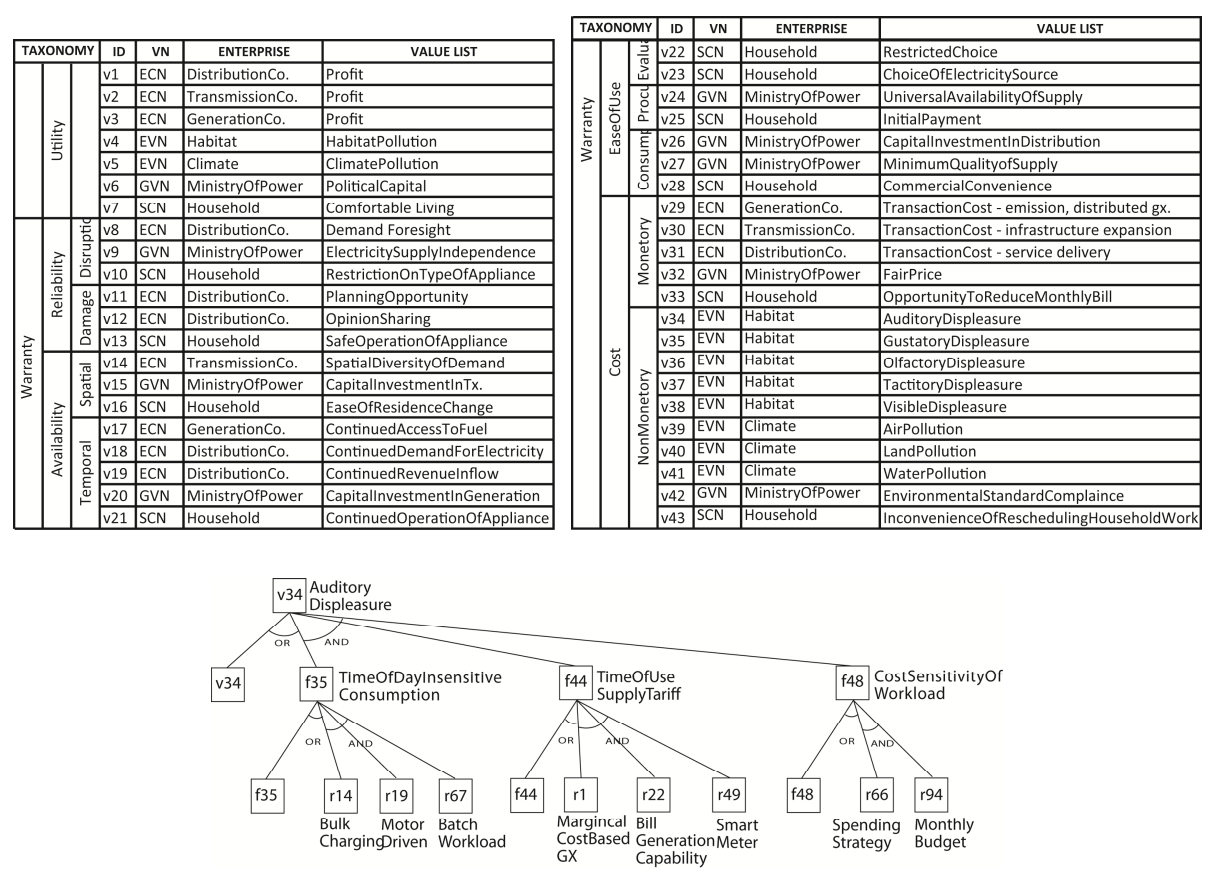

Fig. 9. An Example: Monitoring Auditory Displeasure

The temptation to move workloads to hours of low overall activity, e.g. night time, may result in increased noise levels during odd hours leading to the realization of a negative value of auditory displeasure (v34) to surrounding neighborhoods, the habitat. Use of REGENT to formally represent the value realization process exposes the industry concepts that enable it and the relationship these concepts have with the real world. Industry regulators can use this knowledge, for instance, to clearly identify the different industry elements that need to be monitored so as to track the realization of a given value of interest. An AND/OR graph depicting the value realization process for auditory displeasure (v34) is depicted in Figure 9.

\section{$6 \quad$ Related Work}

The role of ontology in formalizing the concepts in a knowledge system is well established. In the context of industry, ontology development has primarily focused on formalizing the domain specificities. The concepts and relationships that occur between entities from different domains have not attracted much ontological attention. E3 value [11] is one of the few attempts to study the value exchange between the stakeholders in an industry. It is, however, restricted to analyzing the economic exchange between companies active in an e-commerce business. Some ontology development has also been recently noticed in understanding regulation, for example IPROnto [7] which presents a formalization of the concepts in digital rights 
management. In the Electricity industry power quality measurement related ontology has been presented in PQONT [13].

\section{Conclusions}

REGENT enables an explicit specification of multi-party relationships in an industry by formalizing the concepts that influence the realization of stakeholder value. A systematic representation of industry knowledge will expose any deficiencies in regulators' understanding of the industry, thereby assisting the regulator in developing a holistic view of the industry. REGENT is an important first step in our larger effort of developing a knowledge system for the regulation of utilities.

\section{References}

1. Alexander, C.: The Timeless Way of Building. Oxford University Press, New York (1979)

2. Baader, F., Calvanese, D., McGuinness, D.L., Nardi, D., Patel-Schneider, P.F.: The Description Logic Handbook: Theory, Implementation, Applications. Cambridge University Press, Cambridge (2003)

3. Baldwin, R., Cave, M.: Understanding Regulation: Theory, Strategy and Practice. Oxford University Press, Oxford (1999)

4. Barney, J.B.: Firm Resources and Sustained Competitive Advantage. Journal of Management, 99-120 (1991)

5. Beer, S.: Diagnosing the System for Organizations. John Wiley, London (1985)

6. Birchler, U., Bütler, M.: Information Economics. Routledge, London (2007)

7. Delgado, J., Gallego, I., Llorente, S., García, R.: On The Move to Meaningful Internet Systems. In: Workshop on Regulatory Ontologies and the Modelling of Complaint Regulations, Catania, Italy (2003)

8. Gamma, E., Helm, R., Johnson, R., Vlissides, J.: Design Patterns: Elements of Reusable Object-Oriented Software. Addison-Wesley, Holland (1995)

9. Gause, D., Weinberg, G.: Exploring Requirements: Quality Before Design. Dorset House Publishing, New York (1989)

10. Planning Commission (Power \& Energy Division): Annual Report on The Working of State Electricity Boards \& Electricity Departments, Government of India (2002)

11. Gordijn, J., Akkermans, J.: Value-based requirements engineering: exploring innovative ecommerce ideas. Requirement Engineering Journal, 114-134 (2003)

12. Gruber, T.: Toward Principles for the Design of Ontologies Used for Knowledge Sharing. International Journal Human-Computer Studies, 907-928 (2003)

13. Küçük, D., Salor, O., Inan, T., Çadırcı, I., Ermis, M.: PQONT: A domain ontology for electrical power quality. Advanced Engineering Informatics, 84-95 (2010)

14. Malaman, R.: Quality of Electricity Supply: Initial Benchmarking on Actual Levels, Standards and Regulatory Strategies. CERC, Milano (2001)

15. OECD: Regulatory Impact Analysis: Best Practice in OECD Countries, Paris (1997)

16. OGC: ITIL, Version 3. The Stationery Office of Government Commerce, London (2007)

17. Porter, M.E.: Competitive Advantage: Creating and Sustaining Superior Performance. Free Press, New York (1985)

18. Queensland Competition Authority: Electricity Distribution: Service Quality Guidelines. Queensland Competition Authority, Australia (2001) 
19. Ramsay, J.: The Real Meaning of Value in Trading Relationships. International Journal of Operations \& Production Management, 549-565 (2005)

20. Shy, O.: The Economics of Network Industries. Cambridge University Press, UK (2004)

21. Smith, A.: Wealth of Nations. Methuen, London (1904)

22. Ontology Editor and Knowledge Acquisition System,

http: / / protege.stanford.edu/

23. US Aid: Measuring the Quality of Electricity Supply in India. US Aid, India (2007)

24. OWL Web Ontology Language, http: / /www.w3 . org/TR/owl-guide/

25. Wegmann, A., Julia, P., Regev, G., Rychkova, I.: Early Requirements and Business-IT Alignment with SEAM for Business. In: 14th IEEE International Requirements Engineering Conference, Delhi, India, pp. 111-114 (2007)

26. Weinberg, G.: An Introduction to General System Thinking. Wiley \& Sons, NJ (1975)

27. World Commission on Environment and Development: Our Common Future. Oxford University Press, Oxford (1987)

28. Zaccour, G.: Deregulation of electric utilities. Kluwer Academic, Boston (1998) 